\title{
Thoracoscopic management of iatrogenic cardiac perforations
}

\author{
T. Langenaeken ${ }^{1}$, M. Van den Berg ${ }^{1}$, A. Kaya ${ }^{1}$, and A. Yilmaz ${ }^{1}$ \\ ${ }^{1}$ Jessa Ziekenhuis vwz
}

February 14, 2022

\begin{abstract}
Aims: Iatrogenic cardiac perforation is an uncommon but potentially fatal complication of invasive cardiac procedures. When non-surgical management fails, urgent cardiac surgery is required. Standard surgical approach is usually through full sternotomy. However, we propose a less invasive and equally effective technique with video-assisted thoracoscopic surgery (VATS). Methods: This single-center retrospective study in a tertiary hospital identified all patients requiring surgical intervention due to iatrogenic cardiac perforation over a period of 5 years. Patients were grouped by surgical approach, being either sternotomy or VATS. Primary endpoints were length of ICU stay, hospital stay, 30-day mortality and all round mortality. Results: 25 patients were identified: 11 in the sternotomy-group and 14 in the VATS-group. Preoperative baseline characteristics were equal. Significant difference was found for 30-day mortality $(\mathrm{p}<0.05)$. There was no difference for the other endpoints. Conclusions: Videoassisted thoracoscopic surgery is a promising alternative to standard sternotomy for iatrogenic cardiac perforations after invasive cardiac procedures.
\end{abstract}

Thoracoscopic management of iatrogenic cardiac perforations

T. Langenaeken ${ }^{1}$, MD, M. Van den Berg ${ }^{1}$, MS, A. Kaya ${ }^{1}, \mathrm{MD}, \mathrm{PhD}$, A. Yilmaz ${ }^{1}$, MD

${ }^{1}$ Department of Cardiothoracic Surgery, JESSA Hospitals, Hasselt, Belgium

Adress for correspondence

T. Langenaeken M.D., Department of Cardiac Surgery, JESSA Hospitals Hasselt

Stadsomvaart 11

3500 Hasselt

Belgium

tom.langenaeken@hotmail.com / +32478 $408697 /+3211337101$

Author contributions:

T. Langenaeken: conceptualization, methodology, original draft preparation

M. Van den Berg: data gathering, formal analysis, original draft preparation

A. Kaya: review and editing

A. Yilmaz: review and editing Abstract

Aims : Iatrogenic cardiac perforation is an uncommon but potentially fatal complication of invasive cardiac procedures. When non-surgical management fails, urgent cardiac surgery is required. Standard surgical approach is usually through full sternotomy. However, we propose a less invasive and equally effective technique with video-assisted thoracoscopic surgery (VATS). 
Methods : This single-center retrospective study in a tertiary hospital identified all patients requiring surgical intervention due to iatrogenic cardiac perforation over a period of 5 years. Patients were grouped by surgical approach, being either sternotomy or VATS. Primary endpoints were length of ICU stay, hospital stay, 30-day mortality and all round mortality.

Results : 25 patients were identified: 11 in the sternotomy-group and 14 in the VATS-group. Preoperative baseline characteristics were equal. Significant difference was found for 30-day mortality $(\mathrm{p}<0.05)$. There was no difference for the other endpoints.

Conclusions : Video-assisted thoracoscopic surgery is a promising alternative to standard sternotomy for iatrogenic cardiac perforations after invasive cardiac procedures.

Key words : iatrogenic cardiac perforation - complication - invasive cardiac procedures - cardiac tamponade - pericardiocentesis

\section{Introduction}

Invasive cardiac procedures such as percutaneous coronary interventions and electrophysiology procedures have been well established as an effective treatment for coronary lesions and cardiac arrhythmias. Due to their increasing caseload, the low frequency of procedure-related complications can still be a substantial patient group in high-volume centres(1), especially at times when the use of antiplatelet and anticoagulation medication is increasing (2). Acute tamponade due to cardiac perforation is a life-threatening complication. It has been reported that tamponade complicates $0,09-1,42 \%$ of invasive cardiac procedures in high-volume centres(3). In most cases, the primary management is pericardiocentesis. However, this is insufficient in up to $50 \%$ of tamponade cases(1). When non-surgical management fails, urgent surgical exploration is required. The standard surgical approach is almost exclusively by median sternotomy(4). We propose a less invasive technique by thoracoscopic exploration. The aim of this study is to analyse the benefits of video assisted thoracoscopic surgery (VATS) in the surgical treatment of iatrogenic cardiac perforation compared to sternotomy.

\section{Methods}

\section{Patient group}

From September 2016 until September 2021, surgery registration databases were retrospectively reviewed for perforation during invasive cardiac procedures requiring surgical exploration. Cardiac perforation was defined as the occurrence of a pericardial effusion during or immediately after the procedure, associated with hemodynamic instability requiring surgical exploration. 25 consecutive patients were selected; 9 underwent primary full sternotomy, 14 underwent VATS, 2 were converted from VATS to sternotomy. Baseline characteristics were gender, body mass index (BMI) and age. Primary outcomes were duration of intensive care unit (ICU) stay, duration of hospital stay, pericardiocentesis, cardiopulmonary resuscitation, 30-day mortality and all-round mortality. Cardiopulmonary resuscitation was defined by active chest compressions perioperatively due to loss of hemodynamic output. Pericardiocentesis was defined as percutaneous placement of any form of pericardial drainage preoperatively. 


\section{Surgical method}

Surgical method by sternotomy consisted of urgent full sternotomy followed by opening of the pericardium, central cannulation and surgical exploration followed by closure of the perforation by purse string reinforced by teflon felt.

In the thoracoscopy group, three $5 \mathrm{~mm}$ trocars were placed in either the left or right axillary region based on the most probable site of the perforation, after consultation with the cardiologist. Surgical exploration and inspection after incision of the pericardium was done. Perforation was closed in the same manner, by purse string reinforced by teflon felt.

Connection to the extracorporeal circulation (ECC) was by central cannulation in the sternotomy-group and by femoral cannulation in the thoracotomy-group. Indications for connection to the ECC were hemodynamical instability, excessive blood loss or perforation not accessible on beating heart or with lungs inflated.

\section{Statistical analysis}

Baseline study population characteristics by outcome group were described using proportions for categorical variables and mean $\pm \mathrm{SD}$ for continuous variables. Statistical tests were Mann-Whitney-U, Chi-Square and Fisher's exact test. A significance level of $\mathrm{p}<0.05$ was used. All tests were 2-sided. Statistical analysis was made by SPSSR.

\section{Results}

\begin{tabular}{llll}
\hline Perforation site & Sternotomy & Sternotomy & VATS \\
\hline Left atrium & Left atrium & 5 & 5 \\
Left atrial appendage & Left atrial appendage & 0 & 4 \\
Left ventricle & Left ventricle & 2 & 1 \\
Left internal mammary artery & Left internal mammary artery & 0 & 2 \\
Right atrium & Right atrium & 0 & 1 \\
Right ventricle & Right ventricle & 2 & 1 \\
Right coronary artery & Right coronary artery & 1 & 0 \\
Aorta & Aorta & 1 & 0 \\
Coronary sinus & Coronary sinus & 0 & 1 \\
\hline
\end{tabular}

Over a period of 5 years, a total of 25 patients were recorded: 11 in the sternotomy-group and 14 in the VATS-group. The baseline characteristics of the patients were well balanced. Most perforations occurred at the left atrium $(n=10)$ followed by left atrial appendage $(n=4)$. Least likely places of perforation are aorta, right atrium, coronary sinus and right coronary artery of which we only observed one case each (Table 1 ).

Table 1: Perforation sites

There were no statistical differences in the baseline preoperative characteristics (gender, age, BMI). Table 2 displays the results for the primary endpoints. Significant difference was found for 30-day mortality, which was higher in the sternotomy-group. Mean length of ICU and hospital stay was shorter for the VATS-group, however this difference was not significant at alpha level of 0.05 .

\begin{tabular}{llll}
\hline Results & Sternotomy $(\mathrm{n}=11)$ & VATS $(\mathrm{n}=14)$ & $\mathrm{p}$-value \\
\hline Gender $(M / F)$ & $8 / 3$ & $9 / 5$ & 0.20 \\
Age (years) & $71.7 \pm 5.1$ & $68.9 \pm 9.7$ & 0.94 \\
BMI & $27.4 \pm 3.5$ & $28.3 \pm 2.8$ & 0.81
\end{tabular}




\begin{tabular}{llll}
\hline Results & Sternotomy $(\mathrm{n}=11)$ & VATS $(\mathrm{n}=14)$ & p-value \\
\hline Pericardiocentesis & $0.9 \pm 0.2$ & $0.9 \pm 0.2$ & $>0.05$ \\
CPR & $0.3 \pm 0.4$ & $0.1 \pm 0.2$ & $>0.05$ \\
ICU stay (days) & $4.3 \pm 2.7$ & $3.2 \pm 1.8$ & 0.66 \\
Hospital stay (days) & $13 \pm 6.9$ & $9.8 \pm 5.5$ & 0.58 \\
30-day mortality & $\mathbf{2} \pm \mathbf{0 . 5}$ & $\mathbf{0} \pm \mathbf{0 . 0}$ & $<\mathbf{0 . 0 5}$ \\
All-mortality & $4 \pm 0.5$ & $1 \pm 0.1$ & $>0.05$ \\
\hline
\end{tabular}

Table 2: Statistical analysis

\section{Discussion}

Cardiac tamponade is the most frequent lethal complication after catheter ablation and is an independent predictor for mortality $(5,6)$. Standard non-surgical management after cardiac perforation includes percutaneous pericardiocentesis. The risk of late term tamponade still exists combined with the inherent risks of the procedure such as pleural, liver or cardiac puncture. It gives a false sense of security and prolongs the interval until surgical exploration, which is still necessary in up to $50 \%$ of cases(1,7). This study analysed the feasibility of VATS exploration after cardiac perforation. It concludes that VATS leads to a significantly lower 30-day mortality but there are no differences to all-round mortality, length of ICU or hospital stay. Since this is a novel technique, there is little to no available reference literature.

Thoracoscopy avoids the supplementary burden of a full sternotomy and obsoletes its inherent risks such as wound infection of sternal detachment. In contrast to other authors(8), we believe thoracoscopy is faster than a full sternotomy in a dedicated minimally invasive cardiothoracic centre, allowing to quickly estimate the size of the problem and avoiding a full sternotomy for minor perforations.

Almost all possible perforation sites are thoracoscopically accessible. As stated before, most perforations occur at the left atrium or left atrial appendage, which is easily accessible by left sided thoracoscopy even on the beating heart. Should the damage done exceed the limitations of VATS, conversion to full sternotomy is still possible as happened twice in the sternotomy cohort. One case was converted due to massive right ventricle wall rupture after perforating the interventricular septum. Another case was converted to full sternotomy due to the proximity of the perforation to the right coronary artery, requiring closer inspection and complex reconstruction.

Ischemic cerebral and cardiac damage are among the most common causes of death after ablation(6). Most common reason for prolonged ischemic interval are delayed transport to the operating theatre. Femoral cannulation by percutaneous puncture and Seldinger technique can even be done outside the operating theatre, minimizing this ischemic interval. We experience that the longer the delay, the poorer the neurological prognosis.

Lastly, thoracoscopy is an accessible and reproducible method with minimal economic burden. Its main downside is a steep learning curve, emphasizing the need for proper training in centres dedicated to minimally invasive cardiac surgery.

There are several limitations to be adressed. Firstly, the patient group is rather small and single centre. To our knowledge, there are no other centres with the same approach to these cardiac emergencies. Secondly, patients were not randomised. VATS was only performed when the surgeon capable of it was on call for these emergencies. However, there was no patient preselection for either group, confirmed by the absence of differences in the baseline characteristics. For example, one could argue that the more obese patient would be difficult to explore thoracoscopically or that the more critique patient is preselected for sternotomy. However, the results deny any treatment allocation. Thirdly, the delay between perforation recognition and surgical 
exploration could not be withdrawn from our database, which undeniably correlates with (neurological) outcome.

\section{Conclusion}

Video assisted thoracoscopic surgical exploration for iatrogenic perforations after invasive cardiac procedures is equally effective as full sternotomy. 30-day mortality is significantly lower when compared to standard sternotomy. Key to a good outcome is fast intervention when hemodynamic doubt is present. The danger of underestimating the perforation greatly outweighs the relatively minor risks of thoracoscopic evaluation. We stress that the study population is too small to make a solid statement. Our results are hypothesis-generating. A larger, randomized, multicentre validation study is needed.

\section{Declarations}

\section{Competing interests}

No funds, grants, or other support was received by any of the authors for conducting this study.

\section{Ethics approval}

Publication of this retrospective study was approved by the Ethics Committee Research JESSA Hospitals Hasselt.

\section{Consent for publication}

Written patient's or relative's consent for publication was obtained and can be presented if needed.

References

1. Deneke T, MÜgge A. Treatment of cardiac tamponade during electrophysiology procedures: Do electrophysiologists need more than drainage? J Cardiovasc Electrophysiol. 2009 Mar;20(3):284-6.

2. A O, JE S, MY F, B Z. Contemporary evaluation of the causes of cardiac tamponade: Acute and long-term outcomes. Cardiol J [Internet]. 2016 [cited 2021 Oct 12];23(1):57-63. Available from: https://pubmed.ncbi.nlm.nih.gov/26202654/

3. Adamczyk M, Niedziela JT, Wasilewski J, Zembala MO, Kalarus Z, Gąsior M. Prevalence, management and outcomes of cardiac tamponade complicating 66,812 invasive cardiac procedures: single-center clinical registry. Postępy w Kardiol Interwencyjnej $=$ Adv Interv Cardiol [Internet]. 2021 [cited 2021 Oct 12];17(2):193. Available from: /pmc/articles/PMC8356823/

4. Refaat MM, Hashash JG, Shalaby AA. Late perforation by cardiac implantable electronic device leads: clinical presentation, diagnostic clues, and management. Clin Cardiol. 2010 Aug;33(8):466-75.

5. Cheng EP, Liu CF, Yeo I, Markowitz SM, Thomas G, Ip JE, et al. Risk of Mortality Following Catheter Ablation of Atrial Fibrillation. J Am Coll Cardiol. 2019 Nov 5;74(18):2254-64.

6. Cappato R, Calkins H, Chen S-A, Davies W, Iesaka Y, Kalman J, et al. Prevalence and causes of fatal outcome in catheter ablation of atrial fibrillation. J Am Coll Cardiol. 2009 May;53(19):1798-803.

7. TS T, WK F, ME B, GS R, DL P, JB S. Rescue echocardiographically guided pericardiocentesis for cardiac perforation complicating catheter-based procedures. The Mayo Clinic experi- 
ence. J Am Coll Cardiol [Internet]. 1998 Nov 1 [cited 2021 Oct 12];32(5):1345-50. Available from: https://pubmed.ncbi.nlm.nih.gov/9809946/

8. Bogachev-Prokophiev A, Sharifulin R, Elesin D, Zheleznev S, Pivkin A, Karaskov A. Successful totally thoracoscopic management of a superior vena cava perforation with a pacemaker lead. Hear Case Reports. 2016 Jul 1;2(4):300-2. 